\title{
Diagnosis and Treatment of Helicobacter pylori
}

\section{Mae F. Go, MD}

\author{
Address \\ Gastrointestinal Section, Veterans Administration Salt Lake City Health Care \\ System, 500 Foothill Boulevard (111G), Salt Lake City, UT 84148, USA. \\ E-mail: mae.go@med.va.gov \\ Current Treatment Options in Gastroenterology 2005, 8:163-174 \\ Current Science Inc. ISSN 1092-8472 \\ Copyright $\odot 2005$ by Current Science Inc.
}

\section{Opinion statement}

Helicobacter pylori infection remains a ubiquitous infection, especially in populations with poor socioeconomic conditions. Severe clinical outcomes of chronic infection include peptic ulcer disease and gastric cancer. Consensus meetings have developed guidelines for diagnosis, treatment, and management of $H$. pylori infection and related disorders in various populations. Clear benefits are obtained for $\mathrm{H}$. pylori eradication in peptic ulcer disease and gastric mucosa-associated lymphoid tissue lymphoma. Most authorities agree that first-degree relatives of gastric cancer patients should undergo testing for H. pylori infection. H. pylori eradication in dyspepsia remains controversial. Global investigations continue to identify specific host and bacterial factors that are responsible for $H$. pylori-related inflammatory processes and development of clinical disease. Effective eradication regimens have been identified. The proton pump inhibitor (PPI)-based triple therapies are considered first-line therapy because of high patient compliance and good eradication rates. "Quadruple therapy" with bismuth-metronidazoletetracycline plus a PPI is another first-line therapy with a similar eradication rate. This therapy is preferred in patients with penicillin allergy or prior exposure to clarithromycin. Rescue regimens are being developed because of rising antimicrobial resistance to metronidazole and clarithromycin in H. pylori strains. Emerging rescue combination therapies include furazolidone, rifabutin, and quinolones. These combination regimens are still preliminary and should be reserved for patients who have failed first-line therapies. Vaccine development remains elusive.

\section{Introduction}

The successful culture of Helicobacter pylori infection discovered by Barry Marshall and Robin Warren 20 years ago revolutionized our understanding of many upper gastrointestinal tract disorders. Acid was assumed to be the sole culprit in the etiology of peptic ulcers until the discovery of $H$. pylori. Our understanding of this microorganism has matured so we are beyond the observational studies, and global investigations are focused on effective treatment (eradication) of the infection and elucidation of its role in a multitude of clinical disorders $[1 \bullet \bullet]$. H. pylori infection remains a ubiquitous infection. This bacterial infection can be found in an estimated 50\% of the world's population. It is concentrated in populations with poor socioeconomic conditions where associated diseases remain relatively common.
Virtually everyone infected has chronic active gastritis, but it remains unclear which individuals will develop one of the severe clinical outcomes associated with this infection. Some of the clinical outcomes are peptic ulcer disease, gastric cancer, gastric mucosa-associated lymphoid tissue (MALT) lymphoma, or dyspepsia. The infection is most frequently acquired in childhood and can be transmitted from person to person likely by the gastro-oral and fecal-oral routes. The prevalence of the infection usually reflects a birth cohort effect in developed countries, although the prevalence in many developing countries is often high from childhood throughout adulthood. Factors that likely promote acquisition of the infection include poor socioeconomic conditions, large families, and residence in endemic areas [2,3]. 


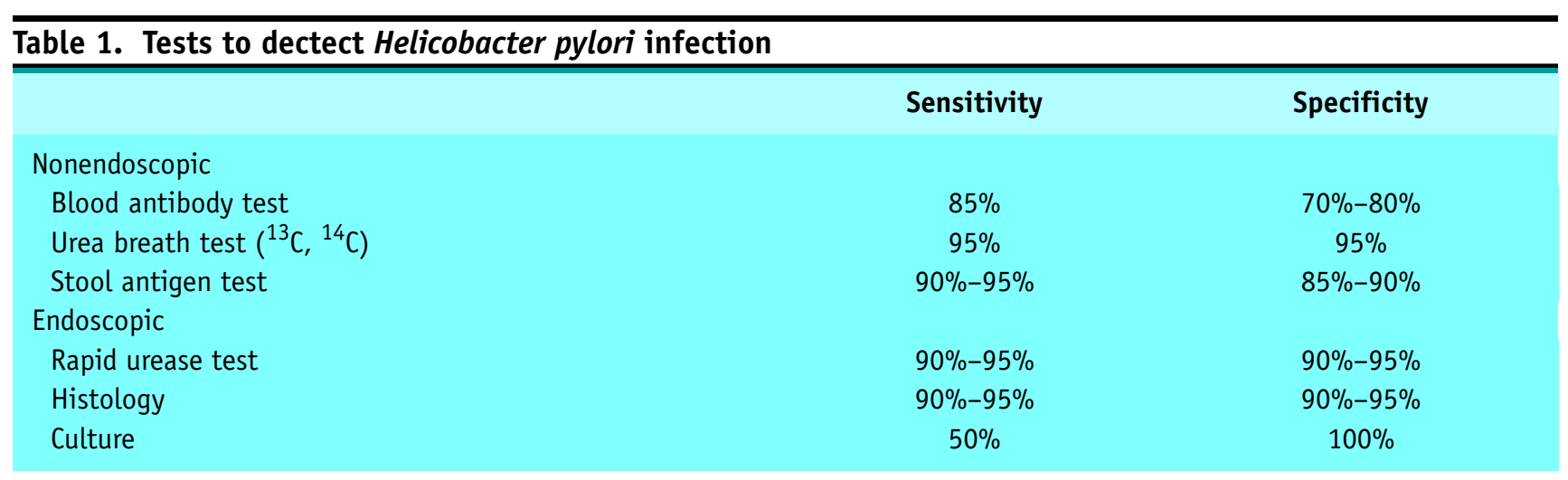

\section{DIAGNOSIS OF HELICOBACTER PYLORI INFECTION}

Eradication of the infection should not be attempted without a positive test result. Eradication treatment should be offered if a positive test result is obtained. Diagnostic tests are well established and widely available (Table 1). Selection of a noninvasive test should be based on prevalence of $H$. pylori infection in the population, past exposure to antibiotics, and availability of the tests [4]. General categories of diagnostic tests for $H$. pylori infection include those that are biopsy-based, utilizing endoscopy (invasive), and those that do not (noninvasive). Noninvasive tests include blood/serum antibody tests, urea breath tests, and the stool antigen test.

Serologic or IgG antibody tests indicate past exposure to the infection; these tests cannot determine whether infection is present. Rapid qualitative immnoassays that can be performed in the office and quantitative enzymelinked immunosorbent assay formats are available. Antibody tests can be very useful in a primary care setting where the prevalence is high and patients have not had frequent exposure to antimicrobial agents that affect $H$. pylori. The sensitivity and specificity of approved antibody tests are $85 \%$ to $90 \%$ and $78 \%$ to $80 \%$, respectively. Because antibodies may remain positive for years after successful eradication and many people have received eradication treatment, the accuracy of serologic tests may now be lower. In general, the antibody test should not be used as follow-up to confirm eradication of the infection. The antibody titer does not help discriminate active or more severe infection. However, laboratory experts suggest that serologic titers can be helpful for long-term follow-up after eradication treatment if the follow-up sample can be compared with the initial diagnostic serum sample. A significant decrease in the titer may be seen 6 months after the infection has been eradicated. Antibodies against $H$. pylori are often present for years after successful treatment, so the presence of blood antibodies does not indicate presence of infection.

Urea breath tests contain either ${ }^{13} \mathrm{C}$ - or ${ }^{14} \mathrm{C}$-labelled urea as the active ingredient. If $H$. pylori infection is present in the stomach, its urease will metabolize the urea to generate excess ${ }^{13} \mathrm{C}$ - or ${ }^{14} \mathrm{C}$-labelled carbon dioxide in collected breath samples. Urea breath tests can determine presence of active infection both before and after eradication treatment. Both types of urea breath tests have been approved by the US Food and Drug Administration (FDA) with an accuracy of 95\% to diagnose and confirm eradication of $H$. pylori infection. The H. pylori stool antigen test is an enzyme-based immunoassay that is also useful for diagnosing active infection [5]. Ideally, the patient should submit a fresh stool to the laboratory. Sensitivity and specificity have been reported at $93 \%$ and $92 \%$, respectively, but some single-center studies suggest that it may be less useful in some populations. European studies indicate that this test is useful for confirming eradication of the infection. Enhanced H. pylori detection has been reported in a European study using a rapid qualitative monoclonal version of the stool antigen test. Pretreatment $H$. pylori sensitivity and specificity were $91.3 \%$ and $93.5 \%$, respectively; post-treatment sensitivity and specificity were $92 \%$ and $100 \%$, respectively [6].

Endoscopic tests for $H$. pylori diagnosis are based upon gastric biopsies obtained from the antrum and/or corpus. These biopsy-based tests can detect active (current) infection. The most cost-effective biopsy-based test is the rapid urease test. Biopsies are placed in a gelatin or filter strip containing urea and a $\mathrm{pH}$ color indicator. When $H$. pylori bacteria are present in the biopsy, the microorganisms will metabolize the urea to generate an alkaline $\mathrm{pH}$-causing a color change. Gastric biopsies for histology are useful if a there is concern about underlying disease or a permanent record is needed. Histology is more expensive than the rapid urease test, but specimens can be reexamined using special stains to confirm diagnoses. Accuracy of the rapid urease test and histology are reported to be about $90 \%$ to $95 \%$. Although culture is typically used to diagnose an infection, it is not recommended for H. pylori. Culture is labor-intensive and many hospital laboratories are not successful in culturing this infection because of its need for specific (microaerophilic) culture conditions, special media, and also because of its slow growth.

It is important to note that accuracy of tests for "active" H. pylori infection will be decreased if the patient is taking proton pump inhibitors (PPIs), high- 
dose H2-receptor antagonists, or antibiotics [7]. These agents can suppress growth of $H$. pylori such that a falsenegative result may be obtained. Authorities recommend "holding" these drugs for 1 to 2 weeks prior to performing the diagnostic test in order to ensure an accurate test result.

\section{GUIDELINES FOR MANAGEMENT OF HELICOBACTER PYLORI INFECTION}

Consensus conferences have been convened in many geographic locations to establish guidelines for diagnosis and treatment of this prevalent infection. These guidelines help provide consistent management approaches, testing for the infection, and recommended eradication treatments to patients with $H$. pylori-associated diseases. The guidelines address issues for the primary care physician and also the specialist. These regional meetings reflect differences in population demographics, $H$. pylori-associated disease burden, economic conditions, and healthdelivery systems. Many of these guidelines are relevant to the US population because of its heterogeneous population. For example, if a patient population includes many immigrants from Asia, Latin America, or Eastern Europe, the frequency of the infection is likely to be greater than in the general US population. These individuals are at greater risk for developing peptic ulcer disease and gastric malignancy in the setting of chronic $H$. pylori infection.

The National Institutes of Health Consensus Panel in 1994 was one of the first consensus conferences to examine the potential role of $H$. pylori in gastrointestinal disorders [8]. This panel recommended testing for the infection in patients with current peptic ulcer disease or a history of peptic ulcer disease. Eradication treatment was recommended for those with a positive test result. Proceedings of the International Update Conference on $H$. pylori confirmed the important role of $H$. pylori in peptic ulcer disease, in gastric cancer, and gastric MALT lymphoma [9]. Practice guidelines from the American College of Gastroenterology have also been published for the US population [10]. The European H. pylori Study Group has held two consensus meetings in which guidelines for their populations are listed [11]. The Japanese Society for Helicobacter Research published guidelines for the diagnosis and treatment of $H$. pylori infection for routine medical practice in Japan [12]. The Latin American Consensus meeting discussed its guidelines within the context of its population because of the variability in the prevalence and clinical outcomes observed in its different member populations [13]. The recent Canadian Consensus conference is particularly pertinent because of its adherence to an evidence-based approach to diagnosis and treatment

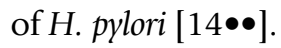

All consensus meetings recommend testing for and eradicating $H$. pylori infection in peptic ulcer disease and gastric MALT lymphoma. The European guidelines recommend $H$. pylori eradication in the patient on chronic PPI therapy due to its potent acid suppression and concern about increased risk for gastric malignancy. Recommendations for eradication of the infection in the setting of gastric hyperplastic polyps, chronic atrophic gastritis, dyspepsia, the patient on chronic PPIs, and postgastrectomy for gastric cancer were variable from region to region. Studies in the Japanese population have confirmed the significant role of $H$. pylori in gastric cancer with recommendations to eradicate the infection to prevent subsequent gastric cancer. The prevalence of gastric cancer has declined in many developed countries with a corresponding uncertainty about screening for this infection to prevent gastric cancer. In those countries where gastric cancer has decreased and is less of a public health burden, few standard guidelines are available. Most authorities agree that firstdegree relatives of patients with gastric cancer should be tested for the infection, and eradication treatment should be offered to those with a positive test result.

\section{HELICOBACTER PYLORI INFECTION AND ASSOCIATED DISORDERS}

Helicobacter pylori is accepted as the most common cause of peptic ulcer in the absence of aspirin or nonsteroidal anti-inflammatory drugs (NSAIDs). A recent meta-analysis confirmed the significant increased risk of peptic ulcer

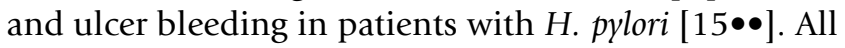
patients with a history of ulcers or a current ulcer should be tested for $H$. pylori infection. Eradication of the infection has resulted in decreased recurrence of peptic ulcer disease [16]. An increasing proportion of peptic ulcers are now found to be H. pylori negative. This is probably due to the combination of the eradication of the infection, the increasing NSAID use, and a decline in $H$. pylori prevalence. This emphasizes the need to confirm $H$. pylori infection prior to prescribing $H$. pylori therapy. If a patient is beginning chronic NSAID therapy and has had a prior ulcer, it is prudent to check for $H$. pylori infection.

There has been some concern that eradication of $H$. pylori infection may cause or exacerbate gastroesophageal reflux disease (GERD) symptoms. Recent studies have shown that many patients have simultaneous peptic ulcer and GERD. Much of the controversy related to this issue is based on the variability in study design, sample size, and endpoint measurements. When $H$. pylori infection is eradicated and the ulcer healed, the GERD symptoms may become apparent. A posthoc analysis of US clinical trials of patients with duodenal ulcer showed no significant differences in erosive esophagitis or GERD after H. pylori eradication [17]. A recent systematic review [18] and a randomized controlled trial [19] concluded that there is little evidence to support an interaction between $H$. pylori infection and GERD. Currently, there is no known causal relationship between GERD and $H$. pylori infection. The impact of $H$. pylori infection in erosive esophagitis heal- 
ing and GERD symptom control were studied in a large European study [20]. Findings from this study indicated that if $H$. pylori infection were present, pantoprazole was more effective in erosive esophagitis healing and symptom control. However, there was no impact of H. pylori in erosive esophagitis in clinical trials with esomeprazole or rabeprazole $[21,22]$. This suggests that there may be differences in acid suppression with different PPIs in the presence or absence of $H$. pylori infection but its overall clinical impact is likely to be small.

Gastric adenocarcinoma remains the second most common cause of worldwide cancer-related deaths. Gastric cancer is often the outcome of a multifactorial process in susceptible individuals with $H$. pylori infection that causes the onset of chronic gastric inflammatory cascade [23]. H. pylori infection was categorized as a Group 1 (definite) carcinogen by the World Health Organization [24]. Seroepidemiologic studies support a four- to sixfold increased risk of gastric cancer in the susceptible individual with chronic $H$. pylori infection [25], although the prevalence of the infection is not a consistent or reliable indicator of the risk within a specific population. Patients with atrophic gastritis who originate from countries such as Japan where gastric cancer remains relatively common are at increased risk for gastric cancer. Uemura et al. [26] have shown that eradication of $H$. pylori can decrease gastric cancer outcome in that population. However, whether or not premalignant gastric histology with intestinal metaplasia and gastric atrophy can be reversed after $H$. pylori eradication with decreased risk remains unclear. Lifestyle and environmental factors as well as host genetic factors are important in this clinical outcome. Studies from El-Omar et al. $[27,28]$ demonstrated that increased interleukin (IL)-1 $\beta$ expression and proinflammatory cytokine profiles are more commonly seen in gastric cancer. The Maastricht meeting has recommended that individu- als taking chronic potent acid-suppressing medications should be tested for $H$. pylori infection because of the potential increased risk of gastric cancer [11].

Helicobacter pylori infection has been implicated in more than $85 \%$ of cases of low-grade gastric MALT lymphoma. This is a rare and indolent malignancy. Partial or complete regression of this tumor has been observed in over $70 \%$ of cases with eradication of the infection. Patients with advanced (high-grade) stages of this malignancy are likely to require surgery and/or chemotherapy.

Whether $H$. pylori infection has a causal role in dyspepsia continues to be debated. Dyspepsia is common and likely related to many different etiologies, as a subset of patients may well have dyspepsia related to this infection [29]. The challenge is identifying patient characteristics or predictors if $H$. pylori infection is implicated in their symptoms. When considering $H$. pylori eradication in dyspepsia, uninvestigated dyspepsia versus nonulcer dyspepsia should be considered [30]. Studies in many primary care settings indicate that the "test and treat" strategy for $H$. pylori infection is effective and reduces cost [31]. A large screening program for $H$. pylori in the United Kingdom showed no statistical benefit in quality of life after eradication in the setting of dyspepsia [32]. No global consensus has been reached about the long-term benefits of $H$. pylori eradication in the patient with nonulcer dyspepsia. This is largely due to variations in the study design, small sample sizes, and differences in follow-up duration and criteria. One meta-analysis showed no benefit for eradication in these patients [33]. A subsequent meta-analysis suggested that there is a small but statistically increased benefit for $H$. pylori eradication in this common clinical disorder [34]. A number of small or single-center studies suggest that eradication of the infection in this population may reduce overall costs by decreasing endoscopy workloads [35]

\section{Treatment}

\section{Diet and lifestyle}

- No particular foods or diet have been shown to eradicate or prevent H. pylori infection. A study of $H$. pylori infection in the Andes suggested that inadequately washed foods were associated with an increased infection rate [36]. Because infection prevalence is increased in lower socioeconomic conditions, it is recommended that foods be washed with clean water. A decline in $H$. pylori prevalence in the general population has been linked to improved socioeconomic conditions with good sanitation and dietary practices. Antioxidant supplements have been suggested because $H$. pylori infection decreases gastric ascorbic acid levels. An intervention study in Latin America showed a similar decrease in premalignant gastric histologic markers using antimicrobial therapy with or without ascorbic acid and beta-carotene [37]. Foods such as garlic, broccoli, and honey have been examined for inhibition of $H$. pylori growth in in vitro studies, but these findings have not been confirmed in human control patients. Probiotics with Lactobacillus have decreased H. pylori loads in humans and animal models $[38,39]$. Some postulate that probiotics 
may be useful as a supplement to antimicrobial therapy. Smoking should be discouraged as it may delay or prolong healing of ulcer disease. Smoking and high dietary salt intake may enhance development of gastric cancer particularly in the setting of H. pylori-induced inflammation [40]. Interestingly, an inverse relationship between alcohol intake and $H$. pylori infection has been observed [41,42]. Laboratory studies suggest that alcohol suppresses growth of $H$. pylori but this has not been prospectively demonstrated in human studies.

\section{Pharmacologic treatment}

\section{Proton pump inhibitors}

Five PPIs are available in the United States; they are omeprazole, lansoprazole, rabeprazole, pantoprazole, andesomeprazole. These prodrugs are the most potent acid inhibitory agents and act by inhibiting the $\mathrm{H}^{+} / \mathrm{K}^{+}$proton pump in gastric parietal cells.

Standard dosage For the "PPI-based triple" regimen, most PPIs are prescribed twice daily. Omeprazole, $20 \mathrm{mg}$ twice daily; lansoprazole, $30 \mathrm{mg}$ twice daily; rabeprazole, $20 \mathrm{mg}$ twice daily; and esomeprazole, $40 \mathrm{mg}$ once daily. No clinical trials using pantoprazole in $\mathrm{H}$. pylori eradication have been performed in the United States, so clinical efficacy in the US population is unproven, but many formularies permit use of pantoprazole, $40 \mathrm{mg}$ twice daily for 10 to 14 days

Contraindications Individual hypersensitivity to the various PPIs appear to be idiosyncratic reactions. Because the duration of treatment using these drugs in eradication treatment is short; there are no major contraindications.

Main drug interactions Interaction of the PPIs with hepatic cytochrome P450 family is a potential source of drug interactions. Omeprazole, lansoprazole, and esomeprazole may induce these enzymes and affect metabolism of other drugs such as diazepam, warfarin, phenytoin, and ketoconazole. Rabeprazole and pantoprazole have the lowest induction of cytochrome P450 and may have the lowest drug interactions. Reported drug interactions with the PPIs are uncommon.

Main side effects Diarrhea, constipation, headache, and abdominal pain

Special points All PPIs are prescribed twice daily except esomeprazole, which is prescribed once daily. FDA-approved duration of treatment is 10 to 14 days except for the rabeprazole-based regimen, which has been approved for 7-day eradication treatment by the FDA.

Cost effectiveness Pricing often depends on formulary with branded PPIs moderately expensive. Branded Prilosec (Procter \& Gamble, Cincinnati, $\mathrm{OH}$ ) is available over the counter. Generic omeprazole available.

\section{Antibiotics}

- Successful treatment (eradication) of H. pylori infection has been proven only with combination antimicrobial treatment. A positive $H$. pylori test result should be obtained prior to prescribing $H$. pylori treatment. Monotherapy should not be considered because no single-drug therapy has effective killing rates of $H$. pylori infection.

- Antibiotics used for first-line eradication treatment include clarithromycin, metronidazole, and amoxicillin. Most treatment durations in the United States have been approved at 10- and 14-day duration. Current FDA-approved combination therapies include two treatment categories (Table 2). First-line treatments include the "PPI-based triple therapy," that consists of a PPI, amoxicillin, and clarithromycin, or "quadruple therapy," that includes bismuth, metronidazole, tetracycline (BMT), and a PPI. Rabeprazole-based triple therapy has been approved by the FDA for 7 -day treatment [43••]. Effective 7-day eradication treatment with esomeprazole, omeprazole, lansoprazole, and pantoprazole has been shown in non-US populations, but no clinical trials using this shorter treatment duration have been performed in the United States. 


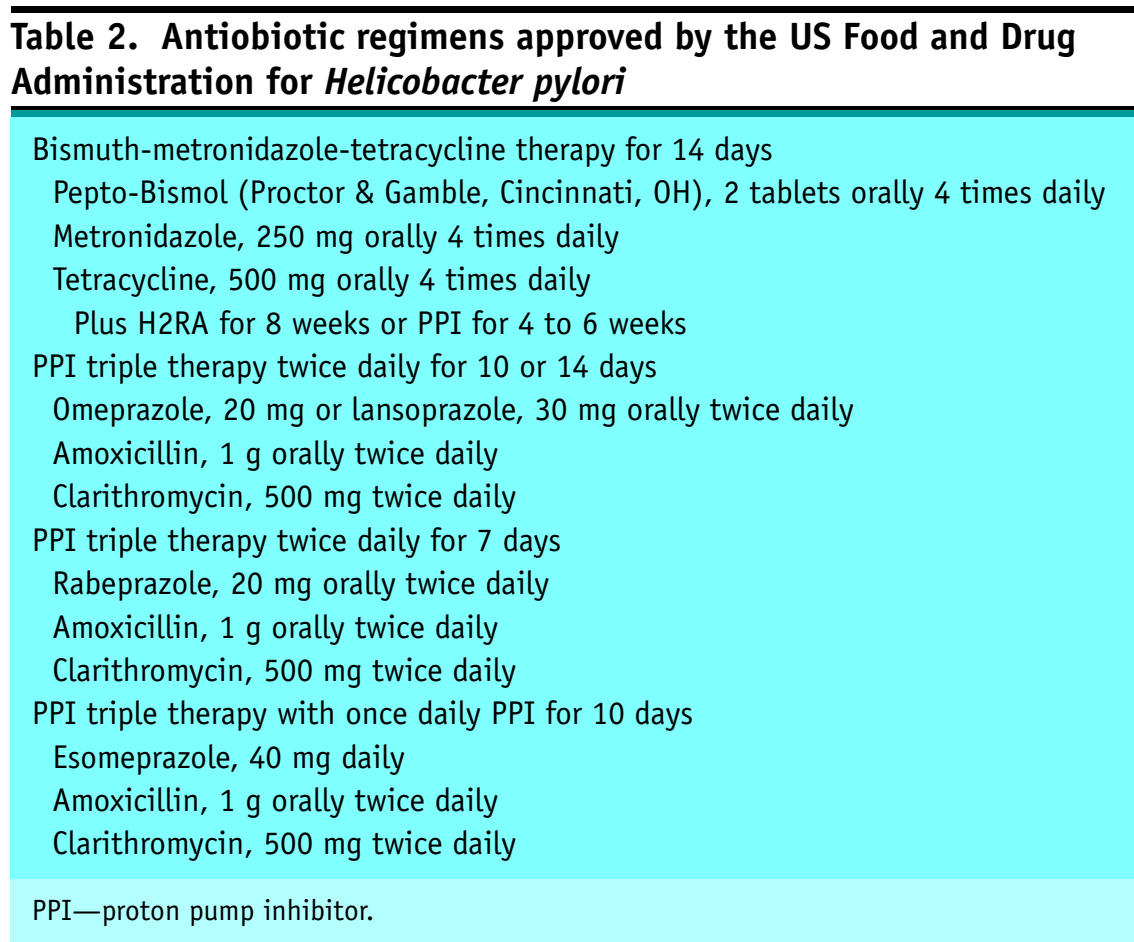

- Proton pump inhibitor-based triple therapy is more frequently prescribed in the United States because of better patient compliance with fewer pills to take. Omeprazole, lansoprazole, and rabeprazole are prescribed twice daily; esomeprazole has received FDA approval for once-daily administration. Regimens have been approved by the FDA for 7, 10, and 14 days with intentto-treat eradication rates of $80 \%$ to $85 \%$ and per protocol eradication rates greater than $90 \%$. PPI triple therapy with clarithromycin and metronidazole can be highly effective for $H$. pylori eradication. Because of the increasing resistance to both these antibiotics, this regimen is not generally recommended for initial treatment.

- The BMT quadruple regimen antibiotics are taken four times daily and this regimen has been approved for 14-day treatment. In the absence of H. pylori antimicrobial resistance, eradication rates are similar among these regimens of greater than $85 \%$ to $90 \%$. If the patient has a penicillin allergy, the BMT quadruple regimen should be prescribed. Other drugs should not be substituted in these approved regimens. For example, azithromycin should not be substituted for clarithromycin. H. pylori treatment trials with azithromycin demonstrate much lower eradication rates compared with clarithromcyin.

A member of the macrolide antibiotics with antibacterial activity based on binding to the $50 \mathrm{~S}$ subunit of the bacterial ribosome and inhibiting protein synthesis. The $500-\mathrm{mg}$

Standard dosage Contraindications

Main drug interactions

Main side effects Special points

Cost effectiveness doses are preferred because lower doses may yield lower eradication rates [44].

$500 \mathrm{mg}$ twice daily.

Hypersensitivity to this drug or other macrolides; avoid concomitant use with cisapride, astemiole, and terfenadine.

Warfarin, statins, phenytoin, rifabutin, and fluconazole.

Metallic taste, abdominal cramping, headache, and dyspepsia.

Patients previously exposed to this medication may be infected by $\mathrm{H}$. pylori resistance to this antibiotic.

Moderately expensive. 
Member of the penicillin family with a broad-spectrum antibacterial activity that inhibit the synthesis of the bacterial cell wall. It is well tolerated and widely available. It should not be substituted with ampicillin.

Standard dosage $1 \mathrm{~g}$ orally twice daily.

Contraindications Do not use in those with penicillin allergy.

Main drug interactions Ethotrexate and allopurinol.

Main side effects Diarrhea, nausea, vomiting, and abdominal pain.

Special points Reports of oral thrush development while on this drug.

Cost effectiveness Available in generic form that is inexpensive.

Metronidazole

\begin{aligned} \hline & Nitroimidazole with antiprotozoal and antibacterial activity. \\ Standard dosage & $500 \mathrm{mg}$ twice daily or $250 \mathrm{mg}$ orally twice daily. \\ Contraindications & Hypersensitivity to metronidazole or other nitroimidazole derivatives; do not use \\ & in first trimester of pregnancy. \\ Main drug interactions & Warfarin. \\ Main side effects & Nausea and abdominal pain. \\ Special points & Potential disulfiram-like interaction of metronidazole and alcohol. \\ Cost effectiveness & Available in generic form that is inexpensive. \end{aligned}

Tetracycline

Inhibits protein synthesis by disrupting RNA translation in microorganisms.

Standard dosage $500 \mathrm{mg}$ orally four times daily.

Contraindications Hypersensitivity to tetracyclines; patients in the last part of pregnancy; children up to 8 years of age.

Main drug interactions Compounds containing aluminum, iron, or calcium may decrease absorption of tetracyclines.

Main side effects Diarrhea, nausea, vomiting, rash, and photosensitivity.

Special points Do not use doxycycline as a substitute.

Cost effectiveness Use generic form that is inexpensive.

Pepto-Bismol

Pepto-Bismol (Proctor \& Gamble, Cincinnati, $\mathrm{OH}$ ) is the only available bismuthcontaining drug in the United States.

Standard dosage $525 \mathrm{mg}$ (2 tablets) orally four times a day.

Contraindications Hypersensitivity to bismuth or salicylates.

Main drug interactions Can interact with warfarin.

Main side effects Constipation, diarrhea, nausea, and vomiting.

Special points Darkening of the tongue and stool; patients should be warned that this is due to the drug and not gastrointestinal tract bleeding.

Cost effectiveness Inexpensive. Available over the counter.

- Factors to consider in the selection of H. pylori eradication treatment include patient compliance, prior exposure to the antibiotics, safety, and allergy. Select a regimen with antibiotics to which the patient has not been previously exposed. If a patient has not been previously treated for H. pylori infection and has had little exposure to antibiotics, either regimen is useful but many practitioners would prescribe the PPI triple therapy because it contains significantly fewer pills. If the patient has been exposed to clarithro- 
mycin, it may be prudent to prescribe the BMT quadruple therapy. If the patient has been previously treated with metronidazole, clarithromycin triple therapy is more likely to be successful. Obviously, if the patient has a penicillin allergy, amoxicillin should not be used. If there is known prolonged exposure to metronidazole and clarithromycin, a metronidazole-containing regimen should be prescribed for a longer duration to "overcome" potential metronidazole resistance.

- Educate the patient in the importance of taking all the prescribed medications to achieve the highest efficacy and to prevent antimicrobial resistance. The patient should be warned about possible side effects because these are multidrug therapies.

\section{Management after a treatment failure}

- Repeat eradication treatment for $H$. pylori infection should be guided by previously administered treatment regimens. Prior exposure to antibiotics may increase the risk of treatment failure if the same antibiotics are used [45]. Bacterial resistance to metronidazole and clarithromycin increases significantly in patients who have failed previous eradication treatments. Antimicrobial resistance in $H$. pylori includes genetic and metabolic processes. Recent estimates include clarithromycin resistance of $10 \%$ to $12 \%$ and metronidazole resistance of $30 \%$ to $40 \%$ in the US adult population [46]. Clarithromycin resistance in $H$. pylori is strongly correlated to treatment failure. Clarithromycin resistance is based on point mutations of the 16S RNA. By contrast, although metronidazole resistance is much more prevalent, resistance is based on $H$. pylori factors such as the RDX gene and appears to be "overcome" by repeat or longer duration regimens that include metronidazole.

\section{Testing for Helicobacter pylori infection after eradication treatment}

- Tests to confirm eradication include the urea breath, the H. pylori stool antigen, biopsy urease test, and histology. Follow-up testing should be undertaken no sooner than 4 to 6 weeks after completion of treatment. As mentioned above, patients should not be on acid-suppressing drugs such as PPIs or high-dose $\mathrm{H}_{2-}$ receptor antagonists or antibiotics because these drugs can suppress the density of $\mathrm{H}$. pylori infection in the stomach. This decreased density can result in a false-negative test result with the urea breath test, stool antigen test, and all biopsy-based tests. Confirmation of eradication is important in complicated ulcer disease. There are no guidelines about follow-up testing in other $H$. pylorirelated disorders. Some authorities recommend follow-up testing in all treated patients, particularly because diagnostic tests have become widely available and are much less expensive.

- Increased treatment failures related to noncompliance and antimicrobial resistance are pushing the search for new antibiotic combinations. A multicenter randomized trial evaluated a "single-triple" capsule of BMT plus omeprazole $[47 \bullet \bullet]$. Omeprazole, $20 \mathrm{mg}$ twice daily plus three oral BMT (OBMT) capsules four times daily was compared with omprazole, amoxicillian, clarithomycin (OAC) twice daily for 10-day treatment; modified intentto-treat eradication rates were $87.7 \%$ for OBMT and $83.2 \%$ for OAC $(95 \%$ $\mathrm{CI}, 3.9 \%$ to $12.8 \% ; P=0.29$ ). Compliance was high. OBMT eradicated $91.7 \%$ 
of $H$. pylori strains that were metronidazole sensitive and $80.4 \%$ metronidazole-resistant strains. OAC eradicated $92.1 \%$ of strains that were clarithromycin sensitive; only $21.4 \%$ with clarithromycin resistance were eradicated with OAC. This study confirmed that OBMT can "overcome" H. pylori with metronidazole resistance.

Rifabutin is a rifamycin-like antimycobacterial drug. It has been designated as an orphan product for use in the prevention or treatment of disseminated Mycobacterium avium complex disease. Rifabutin has been used in pilot and single-center studies for $H$. pylori eradication in patients who have failed other approved $H$. pylori therapies. Perri et al. [48] used $300 \mathrm{mg}$ daily with pantoprazole, $40 \mathrm{mg}$ twice daily and amoxicillin, $1 \mathrm{~g}$ twice daily for 1-week treatment in 41 patients who had failed other PPI-based triple therapies. Intent to treat and per protocol analyses were similar at $71 \%(95 \%$ CI, $57 \%$ to $85 \%)$ and $74 \%$, respectively. It appeared to be well tolerated with all subjects taking at least $80 \%$ compliance. Pilotto et al. [49] determined rifabutin's minimal inhibitory concentration to be 0.0003 to $0.0097 \mathrm{mg} / \mathrm{mL}$ for 87 different strains. This drug is not available in the United States. It is moderately expensive in countries where it is available [50].

Standard dosage Rifabutin has been prescribed at $300 \mathrm{mg}$ daily and $150 \mathrm{mg}$ daily in $\mathrm{H}$. pylori eradication studies.

Contraindications Hypersensitivity to this drug and hepatic impairment.

Main drug interactions Rifampin is known to induce cytochrome P450 enzymes so similar interactions are considered possible for rifabutin. Increased warfarin metabolism and decreased zidovidine serum concentrations may be observed. Concurrent use with azithromycin or clarithromycin has been associated with an increased risk of neutropenia.

Main side effects Nausea, vomiting, diarrhea, rash, and abdominal pain. Neutropenia and uveitis have been reported.

Furazolidone is a nitrofuran derivative with both antibacterial and antiprotozoan activity. It acts by interfering with bacterial enzyme systems. It is extensively metabolized in the intestines and excreted renally. A US single-center study of 27 patients evaluated 14-day furazolidone combination therapy including furazolidone, $100 \mathrm{mg}$ three times daily; tetracycline, $500 \mathrm{mg}$ three times daily; omeprazole $20 \mathrm{mg}$ daily; and metronidazole, $500 \mathrm{mg}$ three times daily or clarithromycin, $500 \mathrm{mg}$ three times daily depending on antimicrobial susceptibility [51]. Overall eradication rate was $97 \%$, but side effects were common and resulted in discontinuation of therapy by $26 \%$ of patients. Eradication rate of only $72 \%$ was obtained with a PPI-triple therapy including furazolidone, tetracycline, and lansoprazole, $30 \mathrm{mg}$ twice daily. Improved $H$. pylori eradication rates were obtained with higher (200 mg twice daily) doses compared with lower doses (100 mg twice daily) [52].

Standard dosage Available in the United States as a 100-mg tablet. Many small studies of $H$. pylori eradication have been conducted with moderate to good efficacy. It should be reserved for those who have failed first-line treatments.

Contraindications Hypersensitivity and hepatic and renal impairment. Allergic angioedema reactions have been reported. It is structurally similar to nitrofurantoin; similar acute pulmonary reactions have been seen with furazolidone. Hemolytic anemia may occur in those with glucose-6-phosphate dehydrogenase deficiency.

Main drug interactions It has monoamine oxidase inhibitory properties, so it may interact with sympathomimetics, tyramine, and amine-containing foods, such as aged cheese, wine, or smoked/pickled meats.

Main side effects Nausea, vomiting, rash, and abdominal pain.

Special point May darken the urine. A disulfiram-like reaction may occur with alcohol-intake. 
Ofloxacin and levofloxacin have been evaluated in combination therapies for $H$. pylor eradication in patients who have failed prior eradication therapies. Levofloxacin is the S-enantiomer of ofloxacin, and it is the primary isomer for antibacterial activity. Antibacterial activity for the quinolones occurs through inhibition of DNA gyrase activity. An open label study using twice daily doses of ofloxacin, $400 \mathrm{mg}$; amoxicillin, $1 \mathrm{gm}$; and rabeprazole, $20 \mathrm{mg}$ had significantly better eradication rates with 14-day compared with 7 -day therapy (92\% versus $62 \%$, respectively) [53]. Preliminary results of 10-day rescue therapy in 107 patients treated with levofloxacin, $250 \mathrm{mg}$ twice daily; amoxicillin, $1 \mathrm{~g}$ twice daily; and esomeprazole, $40 \mathrm{mg}$ twice daily for 10 days yielded and $87 \%$ eradication rate [54]. Studies of $H$. pylori eradication are still preliminary, and the use of the quinolones should be reserved for those who have failed first-line treatments.

Standard dosage $250-\mathrm{mg}$ and $500-\mathrm{mg}$ doses of levofloxacin are available.

Contraindications Hypersensitivity to quinolones. Hyperglycemia has been reported in diabetic patients.

Main drug interactions Increased prothrombin time with bleeding in patients on warfarin; chelated complexes with aluminum, iron, and zinc compounds may occur.

Main side effects Nausea, diarrhea, and headache.

\section{Vaccine development}

- In regions with a high prevalence of $H$. pylori infection and where clinical outcomes such as peptic ulcer disease and gastric cancer are common, the ideal intervention would be a vaccine. The ideal vaccine should be prophylactic, therapeutic, safe, and well tolerated. However, no commercial vaccine is currently available. The large genetic heterogeneity of the microorganism is one of the challenges delaying development of an effective vaccine. Current investigations are evaluating various vectors, adjuvants, and routes of administration in animal models [55].

\section{References and Recommended Reading}

Papers of particular interest, published recently, have been highlighted as:

- Of importance

-• Of major importance

1.• Suerbaum S, Michetti P: Helicobacter pylori infection. $N$ Engl J Med 2002, 347:1175-1186.

This is an excellent discussion of $H$. pylori infection, its disease relationships, and future challenges with this infection.

2. The Eurogast Study Group: Epidemiology of, and risk factors for, Helicobacter pylori infection among 3194 asymptomatic subjects in 17 populations. Gut 1993, 34:1672-1676.

3. Go MF: Epidemiology and natural history of $H$. pylori. Aliment Pharmacol Ther 2002, 16(Suppl 1):3-15.

4. Vakil N, Vaira D: Non-invasive tests for the diagnosis of $\boldsymbol{H}$. pylori infection. Rev Gastroenterol Dis 2004, 4:1-6.

5. Gisbert JP, Pajares JM: Stool antigen test for the diagnosis of Helicobacter pylori infection: a systematic review. Helicobacter 2004, 9:347-368.

6. Gatta L, Perna F, Ricci C, et al.: A rapid immunochromatographic assay for Helicobacter pylori in stool before and after treatment. Aliment Pharmacol Ther 2004, 20:469-474.

7. Gatta L, Vakil N, Ricci C, et al.: Effect of proton pump inhibitors and antacid therapy on 13C urea breath tests and stool test for Helicobacter pylori infection. Am J Gastroenterol 2004, 99:823-829.

8. NIH Consensus Conference: Helicobacter pylori in peptic ulcer disease. NIH Consensus Development Panel on Helicobacter pylori in Peptic Ulcer Disease. JAMA 1994, 272:65-69.

9. Peura DA: The report of the Digestive Health Initiative International Update Conference on Helicobacter pylori. Gastroenterology 1997, 113 (suppl):S4-S8.

10. Howden CW, Hunt RH: Guidelines for the management of Helicobacter pylori infection. Ad Hoc Committee on Practice Parameters of the American College of Gastroenterology. Am J Gastroenterol 1998, 93:2330-2338.

11. Malfertheiner P, Megraud F, O'Morain C, et al. and the European Helicobacter Pylori Study Group (EHPSG): Current concepts in the management of Helicobacter pylori infection--the Maastricht 2-2000 Consensus Report. Aliment Pharmacol Ther 2002, 16:167-180. 
12. Asaka M, Satoh $K$, Sugano $K$, et al.: Guidelines in the management of Helicobacter pylori infection in Japan. Helicobacter 2001, 6:177-186.

13. Coelho LG, Leon-Barua R, Quigley EM: Latin-American consensus conference on Helicobacter pylori infection. Latin-American national gastroenterological societie affiliated with the Inter-American Association of Gastroenerologie (AIGE). Am J Gastroenterol 2000, 95:2688-2691.

14.••Hunt R, Fallone C, van Zanten SV, et al.: Canadian Helicobacter study group consensus conference: update on the management of Helicobacter pylori-an evidence-based evaluation of six topics relevant to clinical outcomes in patients evaluated for $\mathrm{H}$. pylori infection. Can J Gastroenterol 2004, 18:547-554.

Very current, evidence-based guidelines on $H$. pylori diagnosis, eradication treatment, and management approaches for primary and specialty care.

15.• Huang JQ, Sridhar S, Hunt RH: Role of Helicobacter pylori infection and non-steroidal anti-inflammatory drugs in peptic-ulcer disease: a meta-analysis. Lancet 2002, 359:14-22.

This important study discusses the important roles of $H$. pylori and NSAIDs in peptic ulcer pathogenesis and peptic ulcer bleeding.

16. Hopkins RJ, Girardi LS, Turney EA: Relationship between Helicobacter pylori eradication and reduced duodenal and gastric ulcer recurrence: A review. Gastroenterology 1996, 110:1244-1252.

17. Laine L, Sugg J: Effect of Helicobacter pylori eradication on development of erosive esophagitis and gastroesophageal reflux disease symptoms: a post hoc analysis of eight double blind prospective studies. Am J Gastroenterol 2002, 97:2992-2997.

18. Raghunath AS, Hungin AP, Wooff D, Childs S: Systematic review: the effect of Helicobacter pylori and its eradication on gastro-esophageal reflux disease in patients with duodenal ulcers or reflux oesophagitis. Aliment Pharmacol Ther 2004, 20:733-744.

19. Harvey RF, Lane JA, Murray LJ, et al.: Randomised controlled trial of effects of Helicobacter pylori infection and its eradication on heartburn and gastro-esophageal reflux: Bristol helicobacter project. BMJ 2004, 12:328:1417.

20. Holtmann G, Cain C, Malfertheiner P: Gastric Helicobacter pylori infection accelerates healing of reflux esophagitis during treatment with the proton pump inhibitor pantoprazole. Gastroenterol 1999, 117:11-16.

21. Vakil NB, Traxler BM, Levine D: Symptom response and healing of erosive esophagitis with proton-pump inhibitors in patients with Helicobacter pylori infection. Am J Gastroenterol 2004, 99:1437-1441.

22. De Wit NJ, De Boer WA, Geldof H, et al.: Treatment of gastro-oesophageal reflux disease with rabeprazole in primary and secondary care: does Helicobacter pylori infection affect proton pump inhibitor effectiveness? Aliment Pharmacol Ther 2004, 20:451-458.

23. Huang JQ, Sridhar S, Chen Y, Hunt RH: Meta-analysis of the relationship between Helicobacter pylori seropositivity and gastric cancer. Gastroenterology 1998, 114:1169-1179.
24. International Agency for Research on Cancer: Schistosomes, liver flukes and Helicobacter pylori. In IARC Monographs Programme on the Evaluation of Carcinogenic Risks to Humans, vol 61. France: IARC; 1994.

25. A The Eurogast Study Group: An international association between Helicobacter pylori infection and gastric cancer. Lancet 1993, 34:1359-1362.

26. Uemura N, Okamoto S, Yamamoto S, et al.: Helicobacter pylori infection and the development of gastric cancer. N Engl J Med 2001, 345:784-789.

27. El-Omar EM, Carrington $\mathrm{M}$, Chow WH, et al.: Interleukin1 polymorphism associated with increased risk of gastric cancer. Nature 2000, 404:398-402.

28. El-Omar EM, Rabkin CS, Gammon MD, et al.: Increased risk of noncardia gastric cancer associated with proinflammatory cytokine gene polymorphisms. Gastroenterology 2003, 124:1193-1201.

29. Talley NJ, Silverstein MD, Agreus L, et al:: AGA technical review: evaluation of dyspepsia. American

Gastroenterological Association. Gastroenterology 1998, 114:582-595.

30. Chey WD, Moayyedi P: Uninvestigated dyspepsia and non-ulcer dyspepsia-the use of endoscopy and the roles of Helicobacter pylori eradication and antisecretory therapy. Aliment Pharmacol Ther 2004, 19 (Suppl 1):1-8.

31. Chiba N, Van Zanten SJ, Sinclair P, et al.: Treating Helicobacter pylori infection in primary care patients with uninvestigated dyspepsia: the Canadian adult dyspepsia empiric treatment-Helicobacter pylori positive (CADET-Hp) randomised controlled trial. BMJ 2002, 324:1012-1016.

32. Moayyedi P, Feltbower R, Brown J, et al.: Effect of population screening and treatment for Helicobacter pylori on dyspepsia and quality of life in the community: a randomised controlled trial. Leeds HELP Study Group. Lancet 2000, 355:1665-1669.

33. Laine L, Schoenfeld P, Fennerty MB: Therapy for Helicobacter pylori in patients with nonulcer dyspepsia. A meta-analysis of randomized, controlled trials. Ann Intern Med 2001, 134:361-369.

34. Moayyedi P, Deeks J, Talley NJ, et al.: An update of the Cochrane systematic review of Helicobacter pylori eradication therapy in nonulcer dyspepsia: resolving the discrepancy between systematic reviews. Am J Gastroenterol 2003, 98:2621-2626.

35. Sreedharan A, Clough M, Hemingbrough E, et al.: Costeffectiveness and long-term impact of Helicobacter pylori "test and treat" service in reducing open access endoscopy referrals. Eur J Gastroenterol Hepatol 2004, 16:981-986.

36. Goodman KJ, Correa P, Tengana Aux HJ, et al.: Helicobacter pylori infection in the Colombian Andes: a population-based study of transmission pathways. Am J Epidemiol 1996, 144:290-299.

37. Correa P, Fontham ET, Bravo JC, et al.: Chemoprevention of gastric dysplasia: randomized trial of antioxidant supplements and anti-Helicobacter pylori therapy. J Natl Cancer Inst 2000, 92:1881-1888. 
38. Wang K-Y, Li S-N, Liu C-S, et al.: Effects of ingesting Lactobacillus- and Bifidobacterium-containing yogurt in subjects with colonized Helicobacter pylori. Am J Clin Nutr 2004, 80:737-741.

39. Johnson-Henry KC, Mitchell DJ, Avitzur Y, et al.: Probiotics reduce bacterial colonization and gastric inflammation in H. pylori-infected mice. Dig Dis Sci 2004, 49:1095-1102.

40. Joossens JV, Hill MJ, Elliott $P$, et al.: Dietary salt, nitrate and stomach cancer mortality in 24 countries. European Cancer Prevention (ECP) and the INTERSALT Cooperative Research Group. Int J Epidemiol 1996, 25:494-504.

41. Murray LJ, Lane AJ, Harvey IM, et al.: Inverse relationship between alcohol consumption and active Helicobacter pylori infection: the Bristol Helicobacter project. Am J Gastroenterol 2002, 97:2750-2755.

42. Baena JM, Lopez C, Hidalgo A, et al: : Relation between alcohol consumption and the success of Helicobacter pylori eradication therapy using omeprazole, clarithromycin and amoxicillin for 1 week. Eur J Gastroenterol Hepatol 2002, 14:291-296.

43.• Vakil N, Lanza F, Schwartz H, Barth J: Seven-day therapy for Helicobacter pylori in the United States. Aliment Pharmacol Ther 2004, 20:99-107.

This is the first successful 7-day H. pylori treatment regimen approved by the FDA.

44. Juang JQ, Hunt RH: The importance of clarithromycin dose in the management of Helicobacter pylori infection: a meta-analysis of triple therapies with a proton pump inhibitor, clarithromycin and amoxycillin or metronidazole. Aliment Pharmacol Ther 1999, 13:719-729.

45. McMahon BJ, Hennessy TW, Bensler JM, et al.: The relationship among previous antimicrobial use, antimicrobial resistance, and treatment outcomes for Helicobacter pylori infections. Ann Intern Med 2003, 139:463-469.

46. Meyer JM, Silliman NP, Wang W, et al.: Risk factors for Helicobacter pylori resistance in the United States: the surveillance of $H$. pylori antimicrobial resistance partnership (SHARP) study, 1993-1999. Ann Intern Med 2002, 136:13-24.
47.• Laine L, Hunt R, El-Zimaity H, et al:: Bismuth-based quadruple therapy using a single capsule of bismuth biskalcitrate, metronidazole, and tetracycline given with omeprazole versus omeprazole, amoxicillin, and clarithromycin for eradication of Helicobacter pylori in duodenal ulcer patients: a prospective, randomized, multicenter, North American trial. Am J Gastroenterol 2003, 98:562-567.

This study confirmed that a bismuth-containing combination therapy can eradicate or "overcome" H. pylori strains with metronidazole resistance. It should be noted that omeprazole was prescribed twice daily in this 10-day treatment regimen.

48. Perri F, Festa V, Clemente R, et al.: Rifabutin-based 'rescue therapy' for Helicobacter pylori infected patients after failure of standard regimens. Aliment Pharmacol Ther 2000, 14:311-316.

49. Pilotto A, Franceschi $M$, Rassu $M$, et al.: In vitro activity of rifabutin against strains of Helicobacter pylori resistant to metronidazole and clarithromycin. Am J Gastroenterol 2000, 95:833-834.

50. Gisbert JP, Pajares JM: Helicobacter pylori "rescue" regimen when proton pump inhibitor-based triple therapies fail. Aliment Pharmacol Ther 2002, 16:1047-1057.

51. Graham DY, Osato M, Hoffman J, et al.: Furazolidone combination therapies for Helicobacter pylori infection in the United States. Aliment Pharmacol Ther 2000, 14:211-215.

52. Fakheri H, Merat S, Hosseini V, Malekzadeh R: Lowdose furazolidone in triple and quadruple regimens for Helicobacter pylori eradication. Aliment Pharmacol Ther 2004, 19:89-93.

53. Bosques-Padilla FJ, Garza-Gonzalez E, Calderon-Lozano I, et al.: Open, randomized multicenter comparative trial of rabeprazole, ofloxacin and amoxicillin therapy for Helicobacter pylori eradication: 7 vs 14 day treatment. Helicobacter 2004, 9:417-421.

54. Gatta L, Ricci C, Zulla A, et al.: High eradication rate with a rescue levofloxacin based treatment for Helicobacter pylori [Abstract]. Gastroenterol 2004, 126:A74.

55. Michetti P, Svennerholm AM: Helicobacter pyloriinflammation, immunity and vaccines. Helicobacter 2003, 9 (Suppl 1):31-35. 